\title{
Characterisation of Pulverised Fuel Flow in a Square-shaped Pneumatic Conveying Pipe Using Electrostatic Sensor Arrays
}

\author{
Shuai Zhang ${ }^{1}$, Xiangchen Qian ${ }^{1}$, *Yong Yan $^{1,2}$, Yonghui $\mathrm{Hu}^{1}$ \\ ${ }^{1}$ School of Control and Computer Engineering, North China Electric Power University, Beijing 102206, China \\ ${ }^{2}$ School of Engineering and Digital Arts, University of Kent, Kent CT2 7NT, UK \\ Email: y.yan@kent.ac.uk
}

\begin{abstract}
Square-shaped pneumatic conveying pipes are used in some industrial processes such as fuel injection systems in coal-fired power plants and circulating fluidized beds. However, little research has been undertaken to characterise the gas-solid two-phase flow in square-shaped pneumatic conveying pipes. This paper presents novel non-intrusive electrostatic sensor arrays for measuring pulverised fuel in such pipes. The sensor arrays consist of 12 pairs of strip-shaped electrodes which are uniformly embedded in the four flat pipe walls. Experiments have been conducted on a laboratory scale test rig under a range of conditions. The fuel velocity and flow stability profiles over the whole cross-section of the square-shaped pipe are presented in this paper. Experimental results demonstrate that the proposed non-intrusive electrostatic sensor arrays are capable of characterising the velocity and flow stability profiles of pulverised fuel flow in square-shaped pneumatic conveying pipes.
\end{abstract}

Keywords-pulverised fuel; square-shaped pipeline; electrostatic sensor; flow velocity; flow stability

\section{INTRODUCTION}

In coal fired power plants, pulverised fuel is pneumatically conveyed towards a matrix of burners via a primary air pipe network. The fundamental parameters of gas-fuel flow, such as the particle velocity, solid concentration and flow stability, play an important role in improving thermal efficiency and reducing pollutant emissions. Due to the advantages of simple structure and easy installation, square-shaped pneumatic conveying pipes are applied in some industrial processes such as circulating fluidized beds [1] and thermal power plants in certain countries (e.g. Germany).

Owing to the existence of the time-varying interphase force between the air-solid phases, the dynamics of the flow in pneumatic conveying pipelines is extremely complicated [2]. Therefore, most of the existing methods, such as thermal, ultrasonic, capacitive, radiometric, and optical techniques, have shown fundamental limitations in industrial applications for measuring gas-solid flows, especially the particles conveyed in a dilute phase flow $[3,4]$. Due to the advantages of structural simplicity, robustness and low cost, electrostatic sensors outperform other sensors and thus are extensively utilized in multiphase flow measurement. Electrostatic sensors with ring, arc, bar or other shaped electrodes [5-8] have been used in circular pipelines. However, little research has been conducted on square-shaped pneumatic conveying pipes using electrostatic sensors. The particle dynamic characteristics near the four sharp corners are very complex. Electrostatic sensors with square-shaped electrodes have been investigated both theoretically and experimentally by Murnane et al. [9] and Peng et al. [10]. However, the flow parameters of local particles cannot be sensed by such square-shaped sensors.

This paper presents novel electrostatic sensor arrays for the flow measurement of pulverised fuel in square-shaped pipes. The sensing unit consists of 12 pairs of strip-shaped electrodes which are uniformly embedded in the four flat pipe walls. Experimental tests were undertaken on a laboratory scale test rig under a range of conditions. Velocity profiles and flow stability profiles of pulverised fuel over the whole cross-section are illustrated in this paper. Meanwhile, the flow characteristics of pulverised fuel flow in square-shaped pipelines are discussed.

\section{SENSOR DESIGN AND MEASUREMENT PRINCIPLE}

\section{A. Sensor Design}

The arrangement of the proposed non-intrusive electrostatic sensor arrays is shown in Fig. 1. The sensing unit consists of 12 pairs of strip-shaped electrodes which are uniformly embedded in the four flat pipe walls. Fig. 2 indicates the structure of electrostatic sensor arrays from the side view. Three pairs of electrodes are embedded on one side wall. The electrodes are made of copper and embedded in a printed circuit board (PCB). Each electrode has a length of $15 \mathrm{~mm}$ and a width of $3 \mathrm{~mm}$, and the spacing between the two adjacent electrodes in parallel is $15 \mathrm{~mm}$. The signal conditioning circuits are placed on the other side of the PCB. The current signal from the electrode is amplified and filtered by the conditioning circuits. The cut-off frequency of the filter is set to $18 \mathrm{kHz}$. 


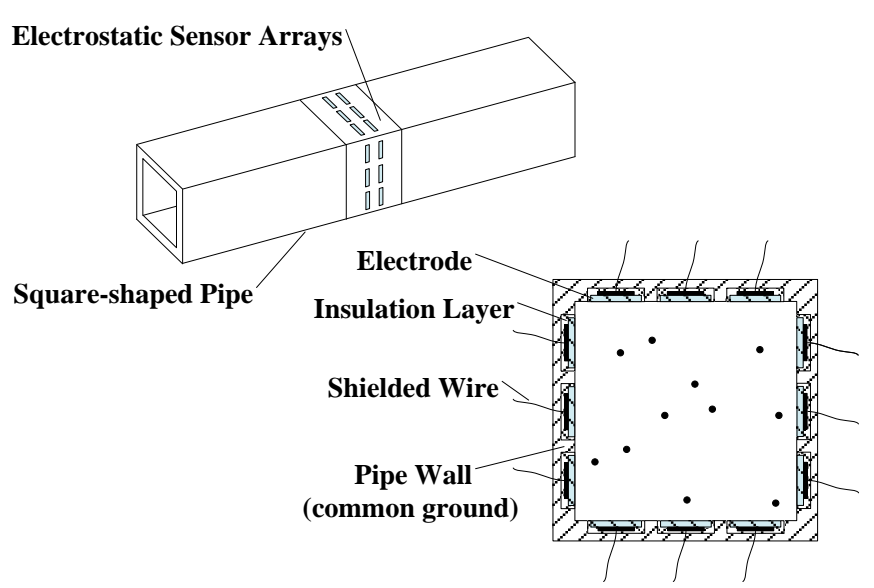

Fig. 1. Physical structure of the electrostatic sensor arrays

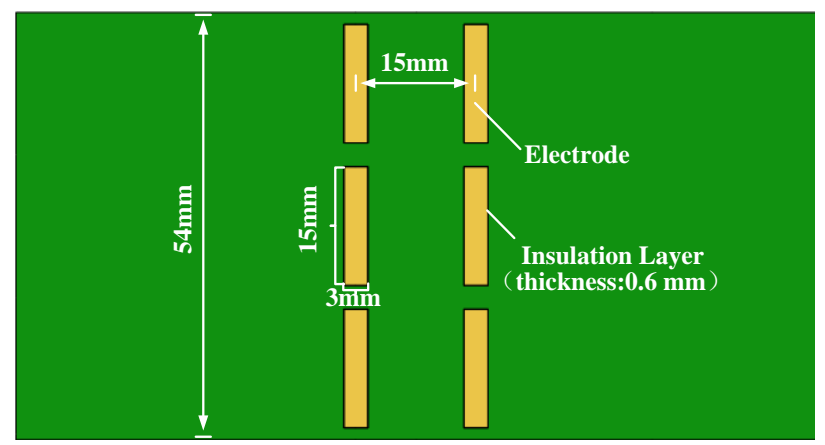

Fig. 2. Electrostatic sensor arrays (on one side)

\section{B. Measurement Principle}

Pulverised fuel particles are charged when conveyed in the pipeline due to the friction with air and the collision between the particles. When the charged fuel particles pass the electrode pairs in sequence, the electrostatic charge on the particles is detected by the sensing unit. Since the two electrodes are identical and adjacent, the signal on the downstream electrode $\left(S_{2}(t)\right)$ is very similar to that on the upstream $\left(S_{1}(t)\right)$ regardless of the time delay [11]. The time delay $\tau_{m}$, i.e. the transit time of particles moving from the upstream electrode to the downstream electrode, is determined from the location of the dominant peak in the cross-correlation function $\left(R_{12}(m)\right)$ of the two signals [12]:

$$
R_{12}(m)=\frac{\sum_{k=0}^{N-1} S_{1}(k) S_{2}(k+m)}{\sqrt{\left.\sqrt{\sum_{k=0}^{N-1} S_{1}^{2}(k)}\right)\left(\sqrt{\sum_{k=0}^{N-1} S_{2}^{2}(k)}\right)}}
$$

where $N$ is the number of the sampling points, $M$ is the maximum number of delayed points $m(m=0,1, \ldots, M), S_{1}(k)$ and $S_{2}(k)(k=0,1,2, \ldots, N-1, \ldots, N+M-1)$ represent the discretization of the signals $S_{1}(t)$ and $S_{2}(t)$ respectively. Since the distance between the two electrodes $(L)$ is given, the correlation velocity is derived from:

$$
v=\frac{L}{\tau_{m}}
$$

The correlation coefficient $r$, i.e. the amplitude of the dominant peak in (1), indicates the similarity of two signals. It can be used to assess the flow stability, i.e. the closer to 1 the correlation coefficient, the more stable the flow near the electrode $[5,7]$.

It should be pointed out that the correlation velocity and coefficient from one electrode pair is not the exact "mean particle velocity" or the "mean flow stability" in the pipe due to the non-uniform sensitivity distribution of the sensor, irregular velocity profile and the difference of correlation computation algorithm [11].

In order to obtain the velocity and stability profiles of the pulverised fuel in square-shaped pipeline over the whole cross-section, the cross section area is partitioned into nine zones, as shown in Fig. 3. Four zones (marked as Zone I, III,VII, IX) are in the corners, four zones (marked as Zone II, IV, VI, VIII) are adjacent to each side wall, and one zone (marked as Zone V) is in the centre of the cross-section.

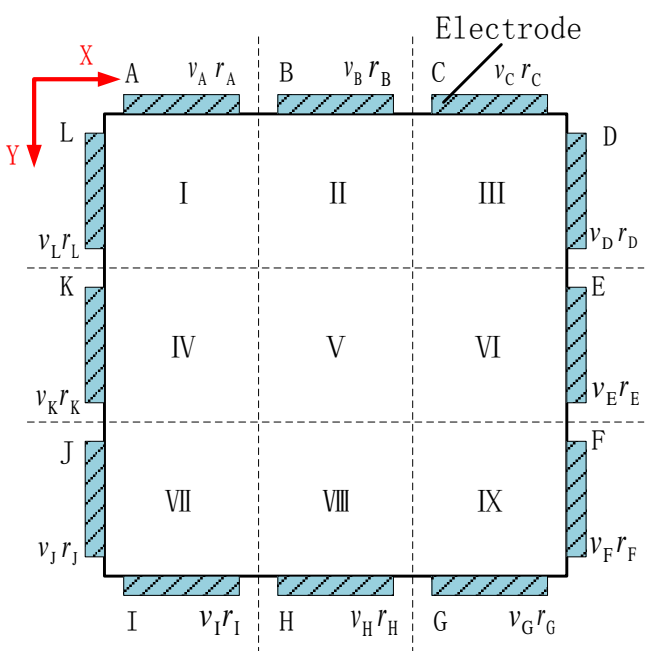

Fig. 3. Area partition on sensor cross-section

For the four corner zones (i.e. Zone I, III, VII and IX), each zone has two pairs of adjacent electrodes. The velocity and correlation coefficient of each zone can be derived from the two adjacent electrode pairs:

$$
\begin{aligned}
& v_{i}=\left(v_{j}+v_{k}\right) / 2 \\
& r_{i}=\left(r_{j}+r_{k}\right) / 2
\end{aligned}
$$

where $i=$ I, III, VII, IX, $j, k=A, L$, or $C, D$, or $F, G$, or $I, J$ accordingly. For Zone II, IV, VI and VIII, the velocity and correlation coefficient of each zone can be presented as the velocity and correlation coefficient of the electrode pair near it:

$$
\begin{aligned}
& v_{i}=v_{j} \\
& r_{i}=r_{j}
\end{aligned}
$$




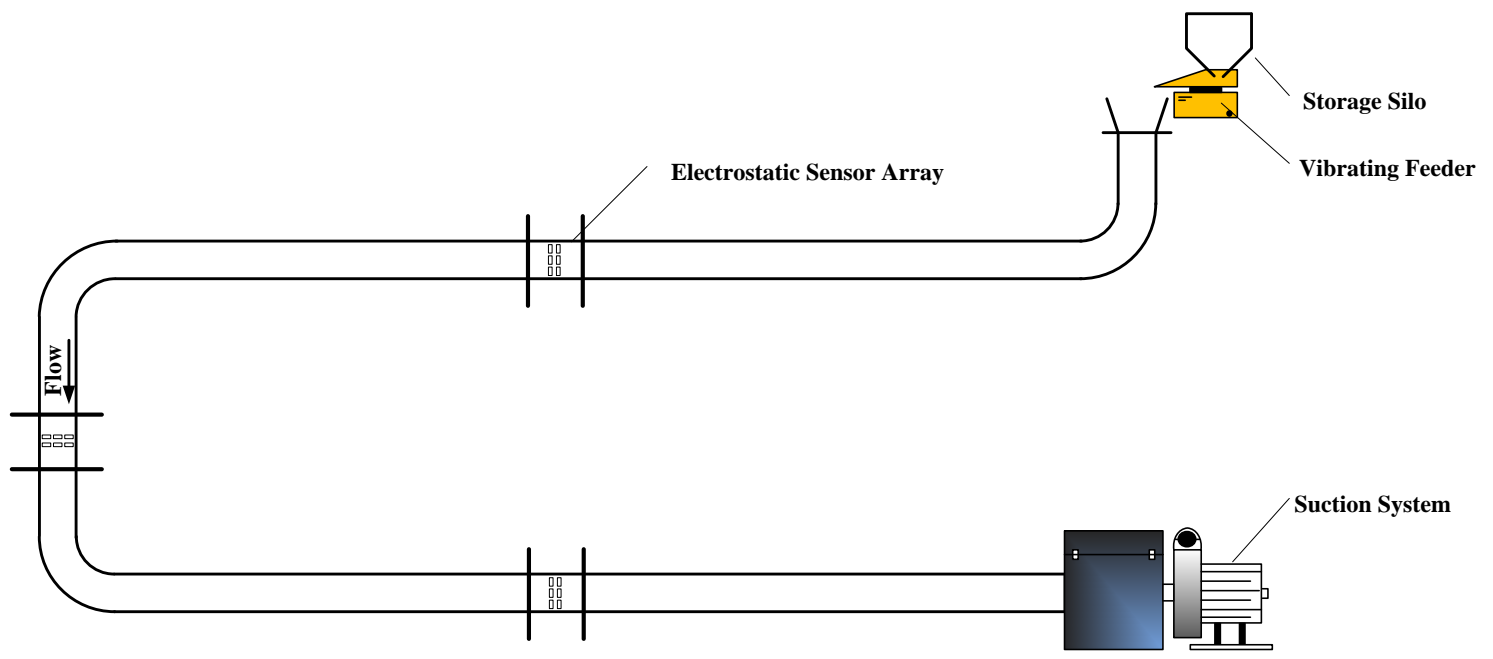

Fig. 4. Layout of the pneumatic conveying test rig (with square-shaped pipe sections)

where $i=I I, I V$, VI, VIII, $j=B, E, H, K$. For Zone V, the centre area of the sensor cross-section, the velocity and correlation coefficient derive from Zone II, IV, VI and VIII, that is:

$$
\begin{aligned}
& v_{\mathrm{V}}=K\left(v_{\mathrm{II}}+v_{\mathrm{IV}}+v_{\mathrm{VI}}+v_{\mathrm{VIII}}\right) / 4 \\
& r_{\mathrm{V}}=K\left(r_{\mathrm{II}}+r_{\mathrm{IV}}+r_{\mathrm{VI}}+r_{\mathrm{VIII}}\right) / 4
\end{aligned}
$$

where $K$ is a dimensionless proportionality depending on the size and layout of the pipeline, of which the value can be obtained through calibration test. Substitution of equations (3)-(6) into equations (7)-(8) yields:

$$
\begin{aligned}
& v_{\mathrm{V}}=K\left(v_{\mathrm{B}}+v_{\mathrm{K}}+v_{\mathrm{E}}+v_{\mathrm{H}}\right) / 4 \\
& r_{\mathrm{V}}=K\left(r_{\mathrm{B}}+r_{\mathrm{K}}+r_{\mathrm{E}}+r_{\mathrm{H}}\right) / 4
\end{aligned}
$$

\section{EXPERIMENTAL RESULTS AND DISCUSSION}

\section{A. Test Rig}

A laboratory-scale test rig (Fig. 4) has been designed and constructed. Stable air flow is generated from an adjustable suction system. Particles are fed to the test sections through an adjustable vibrating feeder. The horizontal square-shaped test pipe section used in this paper is shown in Fig. 5. The inner dimension of the square-shaped pipe section is $54 \mathrm{~mm}$ (Fig. 2).

\section{B. Experimental Procedure and Signals}

Since the size and shape of coal and flour particles are similar [13], flour is used as a substitute of pulverised coal for health reasons within the laboratory conditions. Four groups of experiments were conducted under various working conditions, as shown in Table I. The temperature was $26-27^{\circ} \mathrm{C}$ whilst the relative humidity was $42 \%$ during tests.

A hot wire anemometer (TPI 575) was used to measure velocity of the air in the centre of each zone. The dimensionless proportionality $K$ for this test rig was determined by dividing the velocity of air in Zone $\mathrm{V}$ by the mean velocity of air in Zone II, IV, VI and VIII under four different air velocity conditions. The value of $K$ is 1.07 .

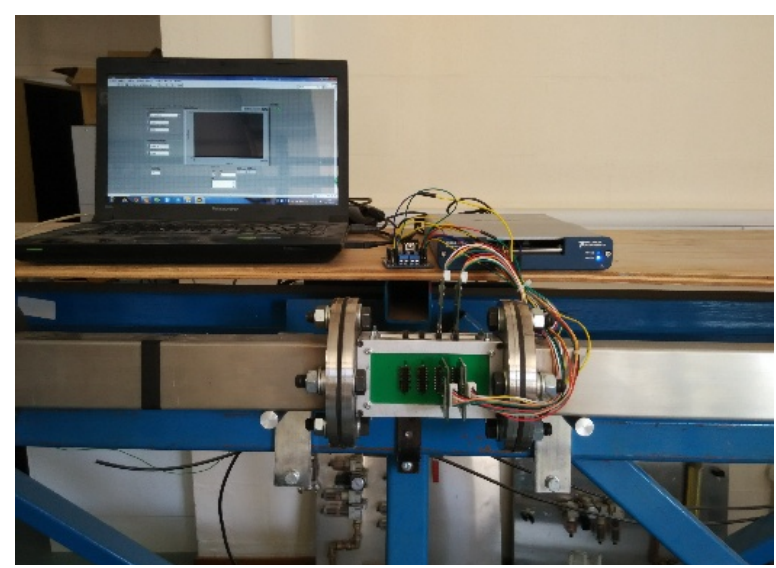

Fig. 5. Horizontal square-shaped test pipe section

TABLE I. EXPERIMENTAL CONDITIONS

\begin{tabular}{|c|c|c|c|c|}
\hline \multicolumn{4}{|c|}{ Mass Flow Rate (kg/h) } & \multirow{2}{*}{$\begin{array}{c}\text { Air } \\
\text { Velocity } \\
(\mathbf{m} / \mathbf{s})\end{array}$} \\
\cline { 1 - 3 } Q1 & Q2 & Q3 & Q4 & \multirow{2}{*}{ Q3.8-14.5 } \\
\hline 5.0 & 10.6 & 14.6 & 18.1 & \\
\hline
\end{tabular}

Fig. 6 shows the electrostatic signal waveforms from a pair of electrodes and the corresponding cross-correlation function. The sampling rate is $50 \mathrm{kHz}$. It is clear from Fig. 6 that the two signals are random but similar. The time delay between them equals to the flow transit time $\tau_{m}$, i.e. the abscissa value of the peak. 


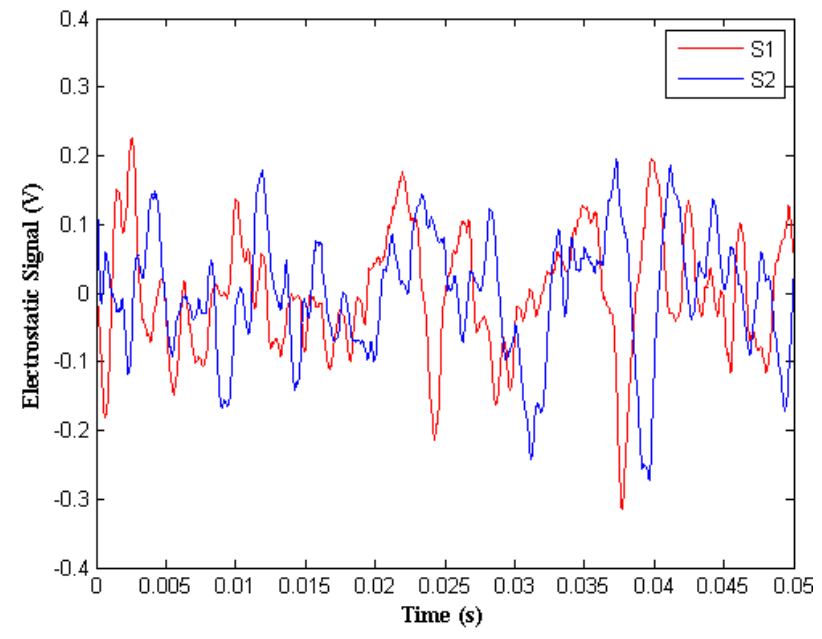

(a) Signals from two electrodes

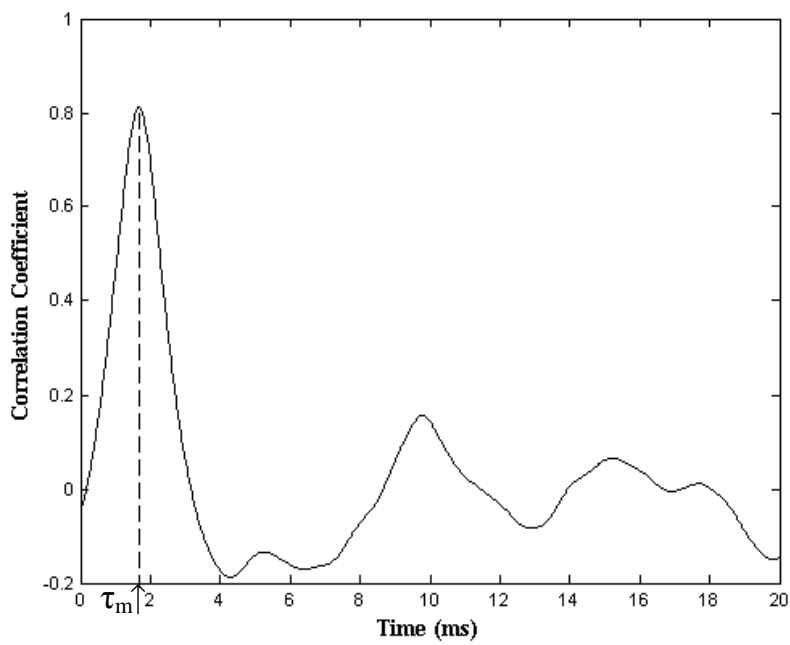

(b) Cross-correlation function

Fig. 6. Signal waveforms of an electrode pair and corresponding cross-correlation function

\section{Results and Discussion}

Each experiment under the same working condition was conducted for three times. 2048 samples from both the upstream and downstream signals were used during each data processing cycle. A total of 1500 velocity and correlation coefficient readings were taken for each test condition (transitional readings were removed). The velocity profile of air measured by a hot wire anemometer (TPI 575) is shown in Fig. 7. The velocity profiles of the particles in nine zones are shown in Fig. 8 to 11. The Zone I, II, III is on the upside and the Zone VII, VIII, IV is on the downside.

Fig. 7 illustrates that the air velocities in the nine zones are not uniform. The velocity of the air in the centre of the pipe (Zone $\mathrm{V}$ ) is the fastest. The air velocity in the four zones (Zone II, IV, VI and VIII) near the pipe wall is faster than those in the four corner zones due to the friction between the air and the wall. By comparing Fig. 8 to 11 with Fig. 7 , we can see that the velocity profiles of the particles over the whole square-shaped cross sections are similar to the air velocity profile, but the particles travel slower. The difference in particle velocities in the nine zones are larger than the difference in air velocity, especially between the corner zones and the central zone as the friction and collision among the air, particles and pipe wall are more complex in the corner zones. However, the difference in velocity reduces as the mass flow rate of particles increases.

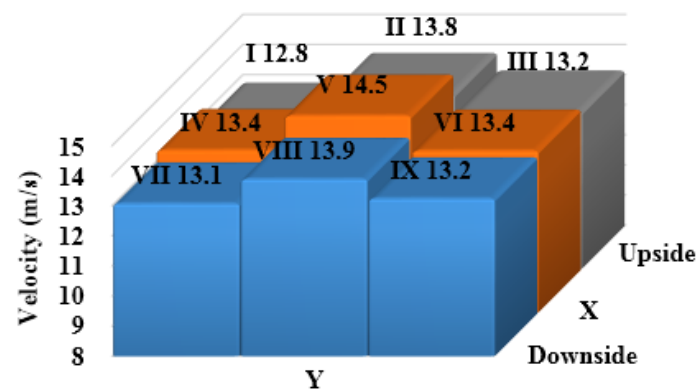

Fig. 7. Velocity profile of air

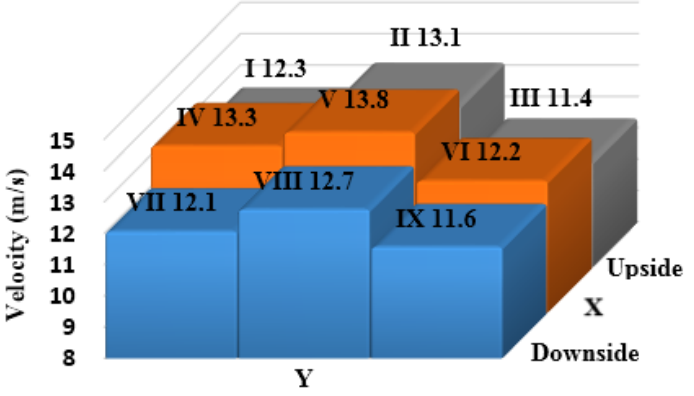

Fig. 8. Velocity profile of particles under mass flow rate Q1

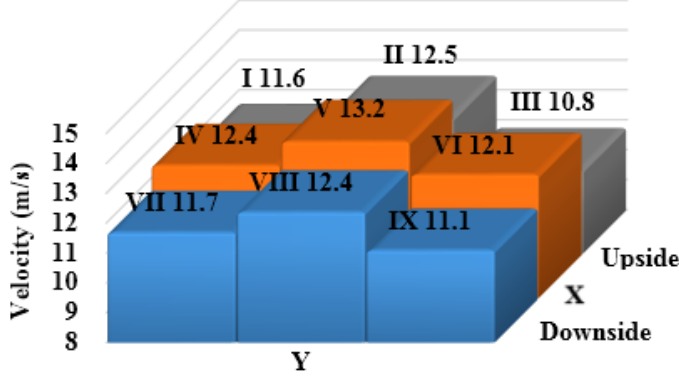

Fig. 9. Velocity profile of particles under mass flow rate Q2

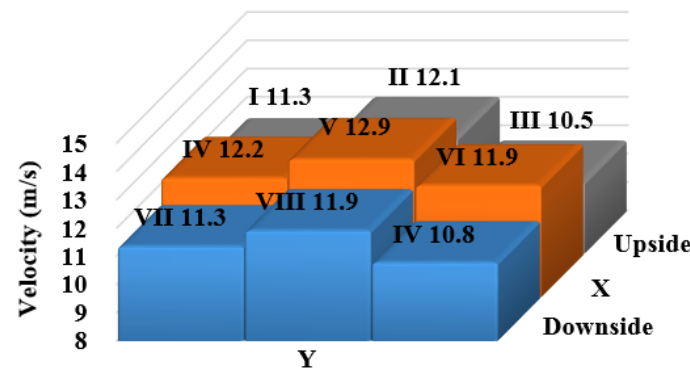

Fig. 10. Velocity profile of particles under mass flow rate Q3 


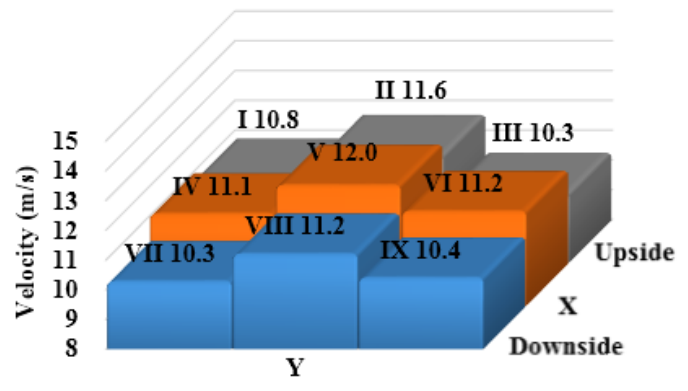

Fig. 11. Velocity profile of particles under mass flow rate Q4

The correlation coefficient profiles of the particle flow under four different mass flow rates are shown in Fig. 12. We can see that the particles in the centre of the pipe (Zone $\mathrm{V})$ move more stably in comparison with the particles in Zone II, IV, VI and VIII near the pipe wall. The flow stability of particles in corner zones (Zone I, III, VII and IX) is the worst owing to the complex dynamics of the flow in corners. It is also observed that a higher mass flow rate results in a more stable flow. This also suggests that the velocity profile becomes more uniform under higher mass flow rate condition.

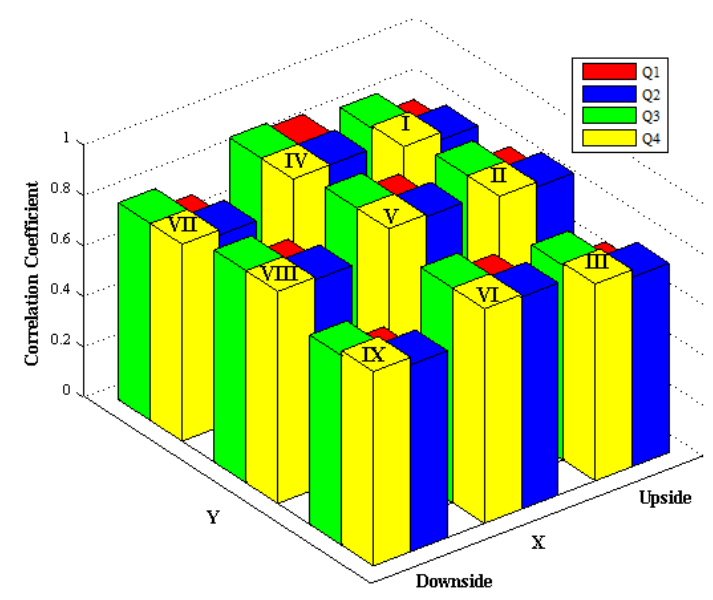

Fig. 12. Correlation coefficient profiles under different mass flow rates

\section{CONCLUSIONS}

Novel non-intrusive electrostatic sensor arrays have been successfully used to measure the velocity and flow stability profiles of pulverised fuel in square-shaped pneumatic conveying pipeline on a lab-scale experimental test rig. The experimental results demonstrate that the fuel in the centre area travels faster and more stably than the fuel along the pipe wall. The velocity of fuel in corners is the slowest and the flow stability is the worst due to the complex dynamics of flow in the corner zones. Further research will be conducted to study the characteristics of the sensor arrays in both mathematical modeling and experiments under a wider range of operating conditions.

\section{ACKNOWLEDGMENTS}

The authors wish to acknowledge the Chinese Ministry of Science and Technology (MOST) and the Chinese Ministry of Education for providing financial support for this research as part of the 973 Project (2012CB215203) and part of the 111 Talent Introduction Project (B12034). This work was also supported by the Fundamental Research Funds for the Central Universities (2014XS46) and the Beijing Municipal Commission of Education.

\section{REFERENCES}

[1] S. Liu, Q. Chen, H. Wang, F. Jiang, I. Ismail, and W. Yang, "Electrical capacitance tomography for gas-solids flow measurement for circulating fluidized beds,” Flow Meas. Instrum., vol. 16, pp. 135-144, 2005

[2] Y. Yan, "Guide to the flow measurement of particulate solids in pipelines,” Powder Handl. Proc., vol. 13, pp. 343-352, 2011.

[3] Y. Yan, "Mass flow measurement of bulk solids in pneumatic pipelines,” Meas. Sci. Technol., vol. 7, pp. 1687-1706, 1996.

[4] Y. Zheng and Q. Liu, "Review of techniques for the mass flow rate measurement of pneumatically conveyed solids," Measurement, vol. 44, pp. 589-604, 2011.

[5] X. Qian, Y. Yan, J. Shao, L. Wang, H. Zhou, and C. Wang, "Quantitative characterization of pulverised coal and biomass-coal blends in pneumatic conveying pipelines using electrostatic sensor arrays and data fusion techniques,” Meas. Sci. Technol., vol. 23, $0853072012,2012$.

[6] J. Krabicka and Y. Yan, "Finite element modelling of electrostatic sensors for the flow measurement of particles in pneumatic pipelines,” IEEE Trans. Instrum. Meas., vol. 58, pp. 2730-2736, 2009.

[7] J.R. Coombes and Y. Yan, "Experimental investigations into the flow characteristics of pneumatically conveyed biomass particles using an electrostatic sensor array,” Fuel, vol. 151, pp. 11-20, 2014.

[8] C. Xu, J. Li, and S. Wang, “A spatial filtering velocimeter for solid particle velocity measurement based on linear electrostatic sensor array,” Flow Meas. Instrum., vol. 26. pp.67-78, 2012.

[9] S.N. Murnane, R.N. Barnes, S.R. Woodhead, and J.E. Amadi-Echendu, "Electrostatic modelling and measurement of airborne particle concentration,” IEEE Transac. Instrum. Meas., vol. 45, pp.488-492, April 1996.

[10] L. Peng, Y. Zhang, and Y. Yan, "Characterization of electrostatic sensors for flow measurement of particulate solids in square-shaped pneumatic conveying pipelines,” Sensor Actuat. A-Phys, vol. 141, pp. 59-67, 2008.

[11] Y. Yan, B. Byrne, S. Woodhead, and J. Coutlhard, "Velocity measurement of pneumatically conveyed solids using electrodynamic sensors,” Meas. Sci. Technol. Vol. 6, pp. 515-537, 1995.

[12] L. Wang, Y. Yan, Y. Hu, and X. Qian, "Rotational speed measurement through electrostatic sensing and correlation signal processing,” IEEE Transac. Instrum. Meas., vol. 63, pp. 1190-1198, 2014.

[13] X. Qian, X. Huang, Y. Hu, and Y. Yan, "Pulverized coal flow metering on a full-scale power plant using electrostatic sensor arrays,” Flow Meas. Instrum., vol. 40. pp.185-191, 2014. 